\title{
Hall Effect Measurements on p-n-p InP Structures
}

\author{
C. A. C. Sequeira and D. M. F. Santos \\ Department of Chemical and Biological Engineering, Instituto Superior Técnico, \\ Technical University of Lisbon (TU Lisbon), Avenida Rovisco Pais 1, 1049-001 Lisboa, Portugal
}

Received on 7 November, 2007

\begin{abstract}
The electrical properties of p-type layers of indium phosphide (InP), formed by the diffusion of zinc into n-type material, are studied by Hall Effect measurements. A wide range of diffusion conditions are used and both homogeneously doped specimens and those containing a zinc atom concentration gradient are produced. A non-correspondence of atom and carrier concentrations is indicated, confirming previous four point resistivity studies. Carrier profiles are achieved by both serial sectioning and multiple specimen techniques. Contacting procedures are developed from which plots of carrier mobility versus carrier concentration, in the range $5 \times 10^{17}$ $-5 \times 10^{19} \mathrm{~cm}^{-3}$, are produced for p-n-p InP. In the main, present results showed good reproducibility and conformed to the "rules" of the Van der Pauw technique.
\end{abstract}

Keywords: Indium phosphide; Zinc diffusion; Hall Effect measurements; Impurity profiles; Carrier mobility; Contacting procedures

\section{INTRODUCTION}

The formation of a p-type region in a previously n-type III-V compound semiconductor, by the indiffusion of divalent impurity atoms, is well known. The relationship between the number of introduced impurity atoms and the number of free carriers produced in the p-type region is dependent on the mechanism of diffusion and in particular on the position of the atoms when diffusion is complete.

One of the most studied of these materials is GaAs with $\mathrm{Zn}$ as the diffusant. An approximate "one to one" correlation between atom and carrier concentrations has been reported by many authors [1-10].

However, for the $\mathrm{Zn} / \mathrm{InP}$ system, such a correlation has not been found. In our previous report [11], we discussed relevant previous work on InP, stressing that for high zinc concentrations, the number of free carriers does not match the number of introduced zinc atoms. Some preliminary studies involving p-n junction depth measurements have suggested a confirmation of this effect even at moderate doping levels.

Here, we briefly describe how this and other techniques have been extended to investigate the electrical properties of p-type layers formed by the diffusion of zinc into InP over a wide range of experimental conditions.

In the main, carrier concentrations and mobility values are found from a combination of resistivity and Hall Voltage measurements. The calculated concentrations from a measurement at a crystal surface only provide a true value for the whole layer if the crystal is uniformly doped. If this is not the case, then the value obtained is simply an average over the layer. The use of a serial sectioning technique with repeated measurements through the depth of the layer can lead to a carrier profile with the aid of well established formulae. These relate carrier concentrations to the properties of the layer before and after the removal of a section. Again, the values obtained will only be absolute for very small section thicknesses.

Four-point probe measurements on zinc diffused InP were described previously [11]. In this paper, we report Hall Effect measurements.
The main advantage of this method over the four-point probe is that from one single experiment, values of resistivity, mobility and carrier concentration can be obtained.

As explained in another paper [12], the most satisfactory method of carrying out the Hall Effect measurement is that due to Van der Pauw [13], using the "clover-leaf" specimen illustrated in Fig. 1. This type of specimen is used in all of this work.

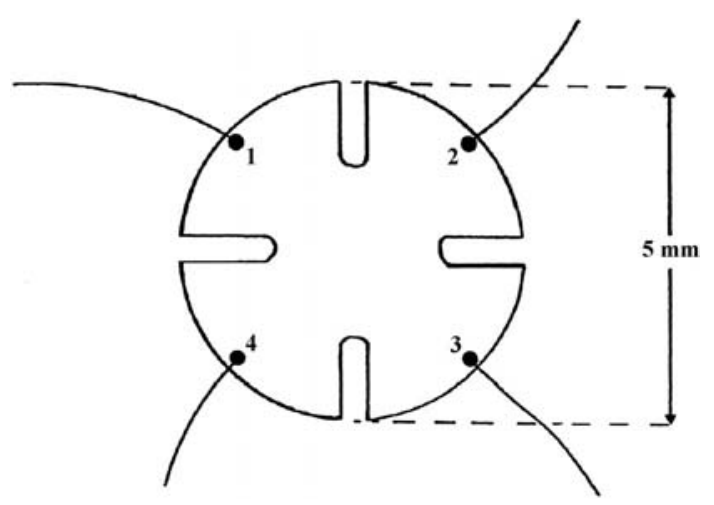

FIG. 1: A "clover-leaf” Hall specimen.

\section{CLOVER-LEAF PREPARATION}

In the course of this investigation, (100) oriented InP wafers, $500 \mu \mathrm{m}$ thick, with a carrier concentration of 6 $10 \times 10^{16} \mathrm{~cm}^{-3}$, and polished to a mirror-like finish, were used. A diffusion specimen was a section of a wafer, about 1 $\mathrm{cm}^{2}$ in area. The wafers were subjected to a standard cleaning procedure (boiling for $10 \mathrm{~min}$. each in $80{ }^{\circ} \mathrm{C}$ trichloroethane, acetone, and methanol; etching for $2 \mathrm{~min}$. in $0.5 \% \mathrm{Br}$ methanol; multiple rinsing in methanol and chloroform). After the cleaning procedure, the wafers were loaded into a clean quartz ampoule containing amounts of phosphorus and nonradioactive zinc which were known, from previous radiotracer experiments, to give a certain profile. In most cases, 
the source contained $1 \mathrm{mg}$ each of zinc and phosphorus. The loaded ampoule was evacuated to less than $10^{-2}$ torr and the diffusion took place in a three-zone furnace accurate to \pm 1 ${ }^{\circ} \mathrm{C}$. The $\mathrm{p}-\mathrm{n}$ junction was made visible by stain etching with $\mathrm{KOH}-\mathrm{K}_{4}\left[\mathrm{Fe}(\mathrm{CN})_{6}\right]-\mathrm{H}_{2} \mathrm{O}$ solution $(1: 2: 6,1.0 \mathrm{~s}$ under intense illumination) and measured with a light microscope. The junction depths obtained varied from about 10 to $160 \mu \mathrm{m}$. For a given set of experimental conditions, the junction lines follow the crystal contours; some of them are straight and flat, others are not flat, and some contain a small number of inclusions.

For the clover-leaf specimen preparation, following diffusion, the crystal of InP was removed from the ampoule and rinsed thoroughly with acetone. If the surface on which the initial measurements were to be taken was not the original diffused surface, then the crystal would, at this point, be etched back to the appropriate depth. Then it was fixed by one of its larger faces to a glass backing plate, using black wax. A mild steel mask cut into the desired clover-leaf design was then placed over the central region of the crystal and secured in place with a further quantity of black wax. The diameter of the mask was $0.5 \mathrm{~cm}$. The completed assembly, then easily handled using the glass plate, was then placed in an S.S. White Industrial Abrasive Unit. In this unit, the areas of wax and semiconductor not protected by the mask were removed using a mixture of $7 \mu \mathrm{m}$ diameter powder and air, directed under pressure through a fine nozzle. Careful manipulation of the nozzle produced a well defined specimen with little or no undercutting beneath the mask. The completed specimen was recovered from the assembly by dissolving away the remaining black wax in trichlorethylene. Since the edges of the original diffused crystal have been entirely removed by the blasting process, no problems are encountered due to the indiffusion of zinc at the side faces. The final clover leaf specimen is a perfect $p-n-p$ structure. Some care is necessary in the handling of these specimens to avoid the fracture of one or more of the four "leaves".

\section{POINT CONTACTING}

The standard contacting technique that has been previously used by many workers is that of surface alloying. Such a technique was tried in this work and the procedure was fairly straightforward. All of the contacts were either of indium or an indium/zinc alloy. The contacting material was provided in wire form and the first step was to cut to small pieces the material from the wire. One piece was then moulded around the end of a short length $(\sim 2 \mathrm{~cm})$ of tinned copper wire and the process repeated four times. The four wires were then positioned, with the indium pieces attached, on the top of the crystal as shown in Fig. 1, ready for alloying. The crystal itself sat on a hot plate inside a small bell jar through which gas could be passed (Fig. 2). With the wires in position, the jar was sealed down and the system flushed out thoroughly with nitrogen gas, for a period of several minutes. The nitrogen was then diverted to flow through a bubbler containing concentrated hydrochloric acid. This provided a flow of $\mathrm{N}_{2}$ plus $\mathrm{HCl}$ vapour through the system. The outgoing gas was bubbled through water to remove the $\mathrm{HCl}$ vapour. After two or three minutes, the hot plate temperature was increased to a point at which the contacting material just melted. At this point, there was a tendency for the indium to form a spherical blob on the surface, but a further increase of temperature to about $200-225{ }^{\circ} \mathrm{C}$ generally caused the blob to collapse and wet the surface around the end of the wire. The temperature was maintained at this level for about 30 seconds and then the specimen was slowly cooled with a flow of pure $\mathrm{N}_{2}$. The presence of $\mathrm{HCl}$ vapour in the gas flow, during alloying, helped in the breakdown of the spherical contact. The final contact was about $0.5-1 \mathrm{~mm}$ across its width.

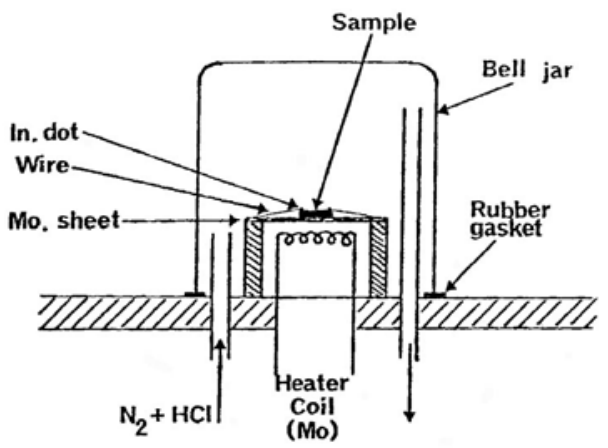

FIG. 2: Rig for alloying contacts.

On n-type material this type of contact proved very successful and manufacture was relatively easy. However, for p-type material, the situation was different. The contacts were often found to be very brittle and easily broken away from the specimen. Also, mechanically sound contacts had non-ohmic electrical characteristics, in general. On certain occasions a good set of contacts could be produced but the reproducibility factor was poor. It has been found [14] that suitable contacts can be made to this type of material using indium/zinc alloys and a more sophisticated system of temperature control. Therefore, one can either use a better temperature programme or change to another system of contacting. The latter of these two alternatives was chosen here because of the type of experiment which was hoped to carry out. This was the production of an accurate carrier concentration profile again by a serial sectioning technique. Not only would alloyed contacts be difficult to remove after measurement on each layer but also the chances of producing 4 good contacts on each of 10 layers, say, is small.

The following tested system used evaporated contacts. These are easy to produce and little damage is done in their removal from a surface. The contacts were $1 \mathrm{~mm}$ dots, several microns thick, formed through a metal mask. Over a period, indium, indium/zinc, aluminium and gold were tried as the contacting material. Evaporation was carried out in a small vacuum system at a pressure of $10^{-6}$ torr. On some occasions, an annealing process was carried out after evaporation, within the vacuum system. Typically, this was for several minutes at $250-350{ }^{\circ} \mathrm{C}$. The final contact to the measuring equipment was made via tinned copper or phosphor bronze wires under 


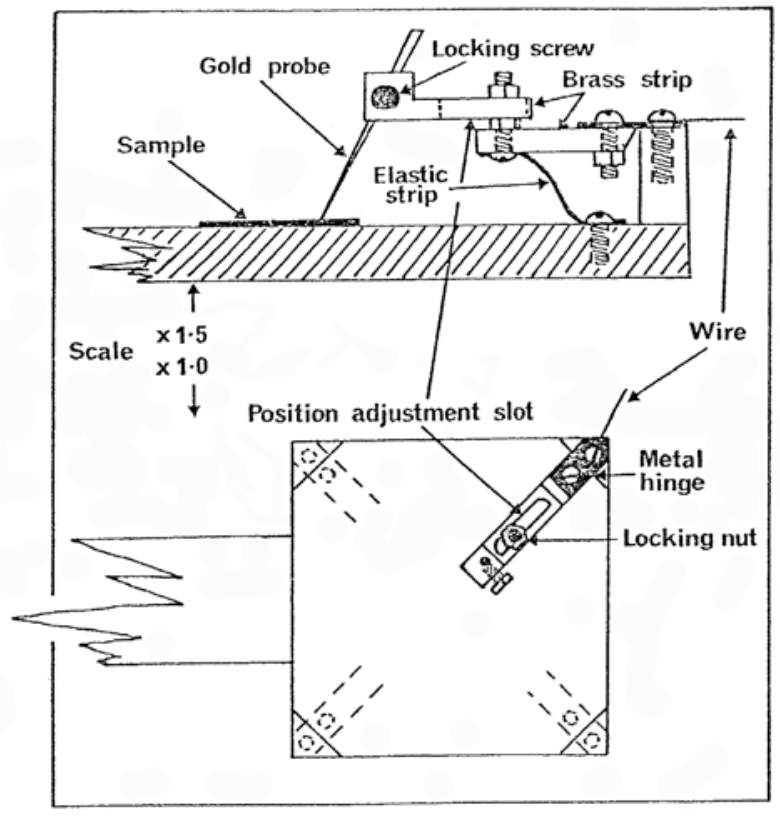

FIG. 3: Hall Effect jig for point contacting.

pressure contact or attached to the dots with colloidal silver paint.

The technique proved to be reasonably successful using indium or indium/zinc dots on n-type material. Other contact materials or the use of p-type specimens led to nonohmic contacts and small values of current flow through the specimen. Some improvement was obtained by annealing the contacts but the method was eventually dropped for measurements on p-type material.

With the experience gained from the four-point probe work [11], a method of point contacting was tried. Such a method satisfies the requirements for easily removable contacts. A small jig (Fig. 3) was built, in order to accommodate the Van der Pauw specimens, with four probes in a square array.

Each probe was held in an independently sprung arm with position adjustment available to eliminate the need for exact specimen positioning. Initially, steel probes were used and gave a similar performance to that found in the earlier work. A switch to gold probes, however, gave an improved performance. For p-type material, "forming" was still necessary but could be achieved at a lower supply voltage $(\sim 15 \mathrm{~V})$. This and the inherently softer gold point led to less surface damage for each run. After each set of measurements, the probes could be simply lifted away from the specimen, which was then etched if required for a layer removal experiment and replaced in the jig. Examples of the type of I-V characteristics found for this type of contact on p-type material before and after "forming" are shown in Fig. 4. Typical contact resistances after "forming" were in the range 20-30 $\Omega$. In comparison, an $\mathrm{I}-\mathrm{V}$ trace for a p-type sample using good alloyed contacts is also shown in Fig. 4.

The correspondence between the traces found for each type of contact is good. As an additional check on the effectiveness of the gold point contacts, Hall Effect measurements were
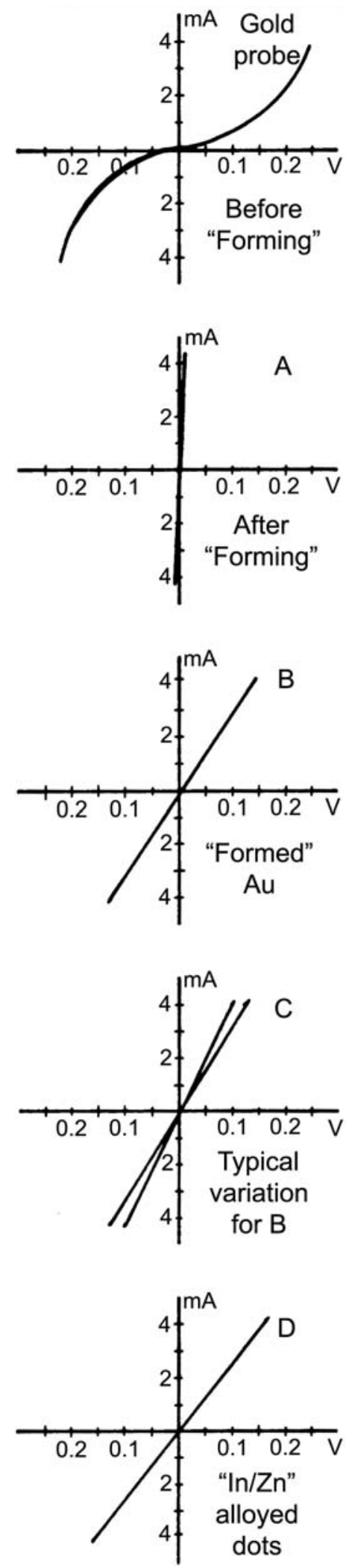

FIG. 4: I-V characteristics on p-type InP. 
TABLE I: Effect of gold contacts on three diffused specimens.

\begin{tabular}{|l|l|l|l|l|}
\hline sample & $\begin{array}{l}\text { carrier concentra- } \\
\text { tion }\left(10^{18} \mathrm{~cm}^{-3}\right)\end{array}$ & $\begin{array}{l}\text { carrier mobility } \\
\left(\mathrm{cm}^{2} \mathrm{~V}^{-1} \mathrm{~s}^{-1}\right)\end{array}$ & $\begin{array}{l}\text { radiotracer atomic } \\
\text { level }\left(10^{18} \mathrm{~cm}^{-3}\right)\end{array}$ & $\begin{array}{l}\text { atom / carrier } \\
\text { ratio }\end{array}$ \\
\hline 700 & 2.3 & 39 & 60 & 26 \\
\hline 900 & 3.5 & 26 & 300 & 85 \\
\hline 750 & 9.5 & 30 & 170 & 18 \\
\hline
\end{tabular}

carried out on three identically diffused specimens; two with point contacting and the other with alloyed contacts. The results are given in Table I and are in good agreement for the values of $\rho, n$ and $\mu$ (see Section IV). Consequently, the point contacting method would seem to be consistent and as good as the more often employed alloying technique. Thus, since it is also particularly suitable for use with successive layer removal, it is used for all of the Hall Effect measurements described in this work.

\section{EXPERIMENTAL PROCEDURE}

After manufacture, the "clover-leaf" specimen was positioned in the jig and the whole assembly placed between the poles of an electromagnet with the specimen surface parallel to the pole faces. Two locating points ensured that each specimen was positioned at exactly the same point within the pole gap. The contacts were then "formed" and their ohmic characteristic checked. The measuring sequence could then begin.

As illustrated in Fig. 1, the coding 1, 2, 3, 4 is used for the different contacts described below. In the Van der Pauw technique, current is passed through the pairs of contacts 12 , 23 and 13 in sequence; voltage measurements being taken across 34,14 and 24, respectively, with no applied magnetic field. A field is then applied and the voltage across 24 remeasured, keeping the current through 13 constant. With no field, $V_{34} / I_{12}\left(\equiv R_{1}\right)$ and $V_{14} / I_{23}\left(\equiv R_{2}\right)$ are a measure of the specimen resistivity. In a perfect case $R_{1}=R_{2}$. However, in practice, due to non-symmetry effects $\left|R_{1}-R_{2}\right|$ is not zero but should equal $R_{3}$ ( $\left.\equiv V_{24} / I_{13}\right)$ if the specimen is of uniform character over its area. This equality condition provides a good check on the validity of each experimental run. Also, $R_{1}$ should be close to $R_{2}$ in value. A correction factor which is a function of $R_{1} / R_{2}$ may be applied, but high values of $R_{1} / R_{2}$ should be suspected. Small cracks, for example, may lead to $R_{1} / R_{2}$ being very large and the value of this ratio is again a good check of the experimental conditions. With a magnetic field applied $V_{24}^{\prime} / I_{23}^{\prime} \equiv R_{3}{ }^{\prime}$ and $\left|V_{24}^{\prime}-V_{24}\right|$ is the Hall Voltage, $V_{H}$.

In this work, for each of the contact pairings, eight separate voltage measurements were taken, the current remaining constant at $5 \mathrm{~mA}$. This was achieved by using both directions of current flow, through 12 say, combined with a switch in polarity between 3 and 4 and then followed by a reversal of the roles of 12 and 34 with current through 34 and voltage across 12 . This switching between sets of contacts and within the pairings was achieved efficiently by using two 6-way switches, linked such that a reading could only be taken if the correct pairings were being used. If any of the contacts were not functioning correctly, it was apparent as a non-equality within the groups of eight readings. For a good experiment all eight readings were similar and an average value was taken.

From these values, the quantities $R_{1}$ and $R_{2}$ were calculated and further work continued only if $R_{1} / R_{2}$ was less than 1.25 . The equality $\left|R_{1}-R_{2}\right|=R_{3}$ was also checked. A $5 \%$ variation was acceptable. Using the Van der Pauw equation [12], the value of bulk resistivity was calculated as,

$$
\rho_{\text {Bulk }}=\frac{\pi b}{2 \log e_{e} 2}\left(R_{1}+R_{2}\right) f\left(R_{1} / R_{2}\right)
$$

where $b$ is the sample thickness. For the case of a diffused layer with a p-n structure, $b$ is taken as the junction depth since the junction provides a region of electrical insulation between the $\mathrm{p}$ and $\mathrm{n}$ regions. $f\left(R_{1} / R_{2}\right)$ has been fully discussed in reference [12]. The sheet resistivity, $\rho_{s}=\rho_{B} / b$, where $\rho_{B}$ is in units of $\Omega \mathrm{cm}$.

The magnetic field used in all of the work was $6 \mathrm{kG}$ produced by a magnet current of $4 \mathrm{~A}$. The magnet was calibrated before starting the work. The residual magnetic field, with no current through the coils was $0.1 \mathrm{kG}$ and had a negligible effect on the "nofield" readings. The specimen was positioned between the poles at all times in order to cut down on the amount of specimen handling. The measurement of Hall Voltage was carried out for both directions of the magnetic field and an average of the two values taken.

The Hall Voltage leads directly to a value of bulk Hall Coefficient, $R_{H}$, and carrier concentration, $\mathrm{p}$, using the equations,

$$
R_{H}=\frac{V_{H} b}{I B}
$$

and

$$
p=\left(R_{H} e\right)^{-1}
$$

where $B$ is the magnetic field and $I$ the specimen current. $R_{H}$ is measured in $\mathrm{cm}^{3} \mathrm{C}^{-1}$ and $p$ in $\mathrm{cm}^{-3}$.

The carrier mobility, $\mu$, is found from,

$$
\mu=\left(p e \rho_{B}\right)^{-1}
$$

and is in units of $\mathrm{cm}^{2} \mathrm{~V}^{-1} \mathrm{~s}^{-1}$.

The use of "one-carrier" equations has been discussed in our previous paper [11], and is valid for the diffused samples used in this work. 
For the case of indiffused n-type specimens or homogeneously doped p-type specimens the values of $\rho_{B}, p(n)$ and $\mu$ will be uniform throughout the depth of the specimen and thus the values calculated using equations (1) to (4) are correct at all points within the specimen and no further calculation is necessary. However, for the case of a diffused layer in which the carrier concentration and mobility are depth dependent, the calculated values of carrier concentration and mobility are weighted averages. For such a layer, an effective number of carriers per $\mathrm{cm}^{3}, N_{s}$, can be defined as

$$
N_{s}=\left(\rho_{B} e \mu_{\mathrm{eff}}\right)^{-1}
$$

with $\mu_{\text {eff }}=R_{H} / \rho_{B}$; the values of Hall Coefficient and bulk resistivity being measured at the top surface of the layer.

In order to eliminate such average parameters, Beuhler [15] devised a set of equations to calculate the values of $p$ and $\mu$ in given layers of a diffused region from information gained from a combination of layer removal techniques and Hall measurements. His equations were,

$$
\frac{\left(R_{s}\right)_{i}}{\left(\rho_{s}^{2}\right)_{i}}-\frac{\left(R_{s}\right)_{i+1}}{\left(\rho_{s}^{2}\right)_{i+1}}=e n_{i} \mu_{i}^{2} d_{i}
$$

and

$$
\left(\rho_{s}\right)_{i}^{-1}-\left(\rho_{s}\right)_{i+1}^{-1}=e n_{i} \mu_{i} d_{i}
$$

which give

$$
\mu_{i}=\frac{\Delta\left(R_{s} / \rho_{s}^{2}\right)_{i}}{\Delta\left(1 / \rho_{s}\right)_{i}}
$$

and

$$
n_{i}=\frac{\Delta\left(1 / \rho_{s}\right)_{i}}{e d_{i} \mu_{i}}
$$

where $R_{s}\left(=R_{H} / b\right)$ and $\rho_{s}\left(=\rho_{B} / b\right)$ are "sheet" parameters, $d_{i}$ is the thickness of the $i^{\text {th }}$ layer and $\Sigma d_{i}=b\left(R_{S}\right)_{i}$ and $\left(\rho_{s}\right)_{i}$ are the quantities found before the removal of the $i^{\text {th }}$ layer and $\Delta\left(R_{s} / \rho_{s}^{2}\right)_{i}$, for example, is the change found in the parameter by the removal of the $i^{\text {th }}$ layer. From the results of this technique, a quantity, $N_{d}$, for the average number of carriers per $\mathrm{cm}^{3}$ can be calculated from,

$$
N_{d}=\frac{1}{b} \sum n_{i} d_{i}
$$

In general, the quantities $N_{d}$ and $N_{s}$ defined above will not be equal. This inequality between the two "average" terms arises because it is not strictly valid to apply the simple Hall Effect equations to the situation of one measurement on a diffused layer. Using these equations, $\mu_{\text {eff }}$ is $R_{H} / \rho_{B}$ and also $\left(N_{s} e \rho_{B}\right)^{-1}$. However, the latter of these terms is actually a conductivity mobility $\left(\sigma=n e \mu_{\sigma}\right)$ and Petritz [16] has shown that the conductivity mobility is weighted by the contribution of layers with higher mobility, in a diffused region, differently to the effective Hall mobility $R_{H} / \rho_{B}$. Therefore, the two expressions for $\mu_{\text {eff }}$ will not actually be equal in practice, leading to $R_{H} \neq\left(N_{s} e\right)^{-1}$. Thus $N_{s}$ is not the true average value of carrier concentration as represented in an equation of the form $R=(n e)^{-1}$. On the other hand, $N_{d}$ is simply an average value calculated from the true concentrations in individual layers and thus is the true average concentration over the whole layer. $N_{d}$ is generally greater than $N_{s}$. In terms of individual layers, Petritz showed that,

$$
\mu_{\mathrm{eff}}=R_{s} / \rho_{s}=\frac{\sum_{i} n_{i} \mu_{i}^{2} d_{i}}{\sum_{i} n_{i} \mu_{i} d_{i}}
$$

whereas

$$
\mu_{\sigma}=\frac{\sum_{i} n_{i} \mu_{i} d_{i}}{\sum_{i} n_{i} d_{i}}
$$

Equations (6) to (9) were used to determine carrier profiles through several diffused specimens which contained atom concentration gradients.

For the initial experiments on homogeneously doped specimens, only one set of measurements for each side of the crystal was required. Reproducibility was checked by removing and replacing the specimen in the jig, with a different orientation. When it was required to study a specimen with a concentration gradient, serial sectioning using chemical etching was employed. This procedure ran into difficulty because of the size and fragility of the Van der Pauw specimens. Even when the etching was done by hand on a polishing pad, breakages were commonplace. Also the small size of the samples led to non-flat etching, with the edges becoming rounded. Some good results were nevertheless obtained with some care. However, it was decided to try and eliminate these problems by encasing the specimens in a resin block, with one face flush to the surface of the block. This should protect the specimen from breakage and also provide a large enough area around the specimen to stop non-parallel layer removal. Of course, in this case the sectioning cannot be accomplished by chemical etching but must be done by mechanical lapping. Unfortunately, this technique was not successful for two basic reasons. Firstly, many specimens suffered from the formation of hairline cracks in the mounting procedure, caused by shrinkage of the resin. Also, it was difficult to assess the amount of material removed in lapping because of the imprecise nature of the block. Later the blocks were mounted in flat and parallel ended cylindrical metal mounts to provide uniformity. Furthermore, it was difficult to position the blocks in the mounts. A dial gauge was used to measure the thickness removed.

The final type of experiment devised for this work eliminated the above problems by the use of several identically diffused crystals. After diffusion, each side of the crystals was etched away to a different predetermined level. This provided several effective depths, through a profile, at the crystal 
TABLE II: Hall Effect measurements on four specimens.

\begin{tabular}{|l|l|l|l|l|l|}
\hline sample & $\begin{array}{l}\text { average resistivity, } \\
\rho_{\text {Bulk }}(\Omega \mathrm{cm})\end{array}$ & $\begin{array}{l}\text { average Hall Coefi- } \\
\text { cient, } R_{H}\left(\mathrm{~cm}^{3} \mathrm{C}^{-1}\right)\end{array}$ & $\begin{array}{l}\text { average carrier concen- } \\
\text { tration, } p\left(\mathrm{~cm}^{-3}\right)\end{array}$ & $\begin{array}{l}\text { average carrier mobility, } \\
\mu\left(\mathrm{cm}^{2} \mathrm{~V}^{-1} \mathrm{~s}^{-1}\right)\end{array}$ & $\begin{array}{l}\text { time } \\
(\mathrm{min} .)\end{array}$ \\
\hline $\mathrm{H} 1$ & $3.40 \times 10^{-2}$ & 1.36 & $4.60 \times 10^{18}$ & 40 & 30 \\
\hline $\mathrm{H} 2$ & $3.07 \times 10^{-2}$ & 1.20 & $5.20 \times 10^{18}$ & 39.2 & 30 \\
\hline $\mathrm{H} 3$ & $3.50 \times 10^{-2}$ & 1.38 & $4.55 \times 10^{18}$ & 39.5 & 30 \\
\hline $\mathrm{H} 4$ & $4.72 \times 10^{-2}$ & 2.00 & $3.13 \times 10^{18}$ & 42.3 & 90 \\
\hline
\end{tabular}

surface (two for each specimen). The Van der Pauw specimens were then cut from the centre of the crystals to provide samples with good flat surfaces. Analysis of the experimental results was also carried out using the Beuhler equations [15] to provide a carrier profile.

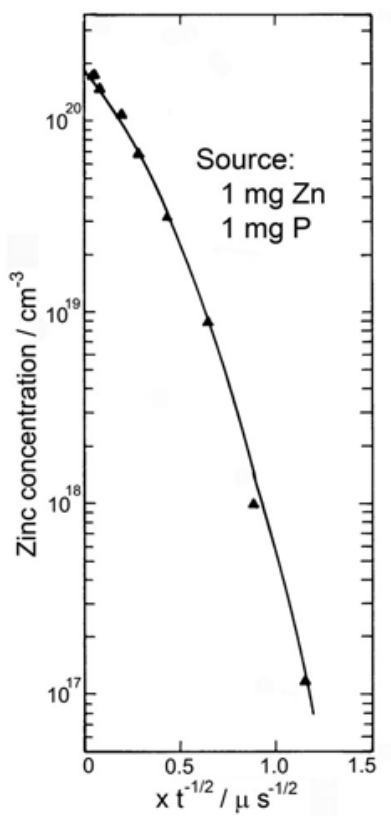

FIG. 5: "Erfc" curve for diffusion of 30 minute duration at $750^{\circ} \mathrm{C}$.

\section{EXPERIMENTAL RESULTS}

Preliminary experiments on "as received" n-type material gave results consistent with the information supplied by Thornton [17]. Carrier concentrations were in the range $6 \mathrm{x}$ $10^{16} \mathrm{~cm}^{-3}$ to $1 \times 10^{17} \mathrm{~cm}^{-3}$. Mobility values were $3400-3600$ $\mathrm{cm}^{2} \mathrm{~V}^{-1} \mathrm{~s}^{-1}$ and bulk resistivity around $3 \times 10^{2} \Omega \mathrm{cm}$. Both indium alloyed contacts and gold probes were used for several different samples. Annealing the n-type material for several hours at $750{ }^{\circ} \mathrm{C}$ did not affect its electrical characteristics at all.

Homogeneously doped samples were prepared by long diffusions at $700{ }^{\circ} \mathrm{C}, 750{ }^{\circ} \mathrm{C}$ and $900{ }^{\circ} \mathrm{C}$, using a $1 \mathrm{mg} \mathrm{Zn} / 1$ $\mathrm{mg} \mathrm{P}$ source. The values of resistivity, mobility and carrier concentration as calculated from equations (1) to (4) are given in Table I. For comparison purposes, the corresponding tracer atomic concentration is lower than the atomic concentrations.
The ratios between the two parameters are 26, 18 and 85 for the 700,750 and $900{ }^{\circ} \mathrm{C}$ diffused specimens, respectively. The mobility values fall with increasing diffusion temperature and atomic concentration. The mobility of the carriers in p-type $\mathrm{InP}$ is seen to be low and a factor of about 100 lower than for electrons in n-type material.

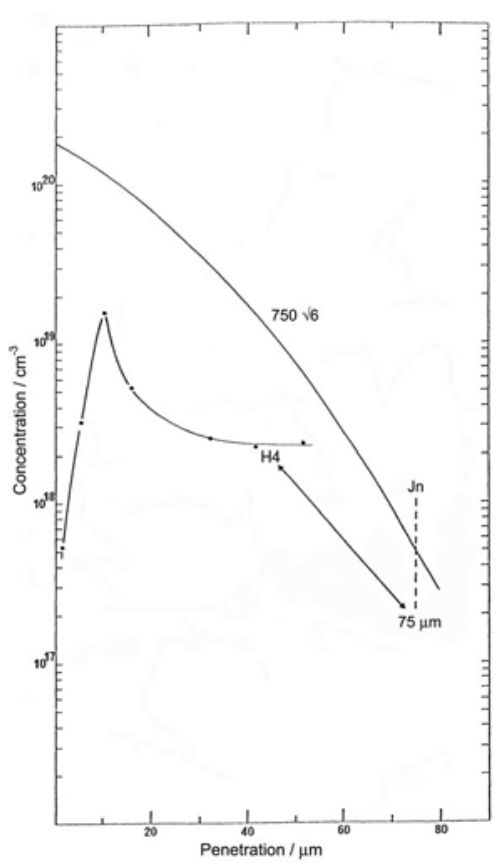

FIG. 6: A carrier profile determined from Hall Effect measurements with serial sectioning. Also included are corresponding tracer profiles, stain junction depths and background donor level.

The first group of diffused layers to be investigated was in crystals diffused at $750{ }^{\circ} \mathrm{C}$ for 30 minutes with a $1 \mathrm{mg} \mathrm{Zn}$ $/ 1 \mathrm{mg} \mathrm{P}$ source. This corresponds to the radiotracer profile shown in Fig. 5. There will be a time factor of $\sqrt{ } 2$ but the "erfc" form may fairly safely be assumed to still exist. Three samples were prepared, two $(\mathrm{H} 1, \mathrm{H} 2)$ for Hall measurements with gold probes and one (H3) for measurement using zinc/indium alloyed contacts. The effective or average values of $\rho_{B}, p$ and $\mu$, as measured at the original surface, are given in Table II for all three samples. Mobility values are typically $40 \mathrm{~cm}^{2} \mathrm{~V}^{-1} \mathrm{~s}^{-1}$ with bulk resistivity around $3 \times 10^{-2} \Omega$ $\mathrm{cm}$. Attempts to serially section these samples were not successful due to the fairly shallow junction and non-flat etching. The average values of $p\left(N_{s}\right)$ are much lower than the corresponding average atomic concentration as estimated from the 
radiotracer profile. Again a figure of 20 is a rough estimate of the atom/carrier ratio.

Better results were obtained under similar conditions but using a longer diffusion time of 90 minutes. With careful etching, it was possible to produce a good set of results by successive layer removal. The thickness of each removed layer varied between 3 and $10 \mu \mathrm{m}$. A typical example is $\mathrm{H} 4$ and the average values of $\rho_{B}, p$ and $\mu$ at the surface of this specimen are also given in Table II. They are very similar to those of the shorter time diffusions (H1, H2). Fig. 6 shows the carrier profile produced by using the calculations of Beuhler [15] on the sectioning and Hall measurements to give $n_{i}$ and $\mu_{i}$ for each layer. Also included is a corresponding "tracer profile". This is in fact the curve of Fig. 5, now with a time factor of 2.45 $(\sqrt{ } 6)$ applied.

This approximation of assuming an "erfc" form still is quite adequate for the purpose of comparison between atom and carrier levels. At all points, the carrier profile is below the "tracer curve". A sharp peak in carrier concentration near the surface is followed in the bulk by a flat region of uniform concentration at about 2-2.5 x $10^{18} \mathrm{~cm}^{-3}$. The $\mathrm{p}-\mathrm{n}$ junction, found by chemical staining, corresponds to an atomic concentration of $5 \times 10^{17} \mathrm{~cm}^{-3}$ on the "tracer profile". A plot of mobility versus concentration $\left(\mu_{i} v s . n_{i}\right)$ for this specimen is shown in Fig. 7. The mobility is seen to decrease with increasing concentration. The effective or average mobility as measured at the original surface of the diffused layer is marked with an arrow. Also plotted in this figure are the mobility values found for the homogeneously doped specimens. For H4, the effective concentration value $N_{s}$ is $3.13 \times 10^{18} \mathrm{~cm}^{-3}$ whereas $N_{d}$ is $4.18 \times 10^{18} \mathrm{~cm}^{-3} . N_{s}$ is therefore about $75 \%$ of $N_{d}$, a figure similar to that found by Mayer [18] in implanted layers of silicon. For all of the p-type layers which were sectioned and probed at successive depths, the transition from p- to n-type material was found to correspond fairly closely $(\sim 3 \mu \mathrm{m})$ to the position of the $\mathrm{p}-\mathrm{n}$ junction as shown in the staining procedure. This is an important result since two completely different techniques are shown to be in agreement on the position of the p-n junction. The reproducibility of results throughout this type of experiment was good.

From the experiment in which a number of nominally identical samples were used (H5), in order to eliminate the need for sectioning between measurements, a form of analysis similar to that described above was used. Fig. 8 shows the calculated carrier profile and the corresponding "tracer profile" (H5T).

Again, an initial rapid fluctuation in carrier concentration is followed by a flattening out to approach a level of about $4 \times 10^{18} \mathrm{~cm}^{-3}$. The mobility values are plotted in Fig. 7 together with the average mobility as found at the original surface. The stained junction depth for these samples occurs at roughly $48 \mu \mathrm{m}$ corresponding to an atomic concentration of 9 $\mathrm{x} 10^{17} \mathrm{~cm}^{-3}$ (from measurements of $\mathrm{p}-\mathrm{n}$ junction depths and radiotracer profiles not reported in this paper).

As an example, the tabulated results and calculation steps for a Hall (H4) experiment are shown in Table III.

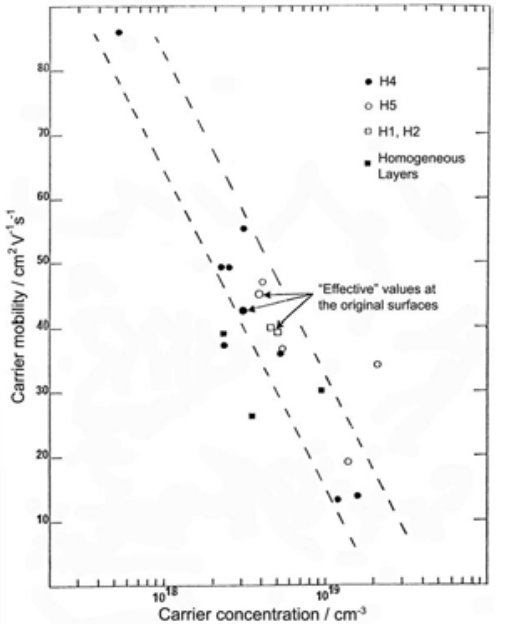

FIG. 7: A plot of mobility versus concentration for the holes in zincdiffused layers of p-type InP.

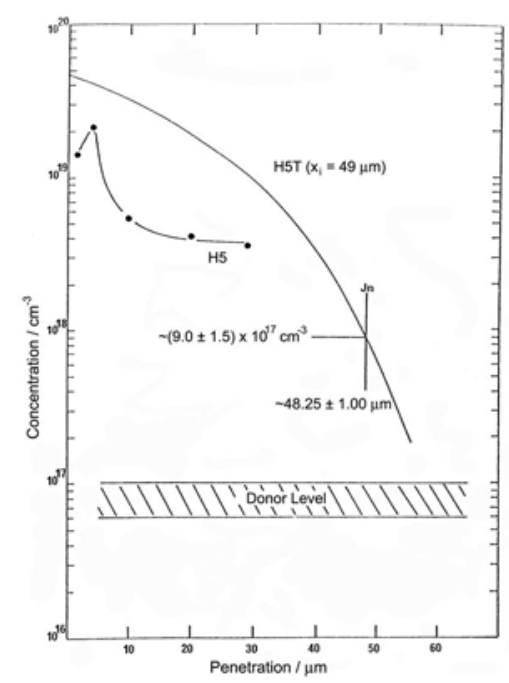

FIG. 8: A carrier profile determined from a multi-specimen Hall Effect experiment with a corresponding tracer profile. Also illustrated are the errors involved in determining the concentration levels at stained junction positions.

\section{DISCUSSION}

In all the experiments described in this paper, one feature of the experimental results is outstanding. This is the fact that in a diffused layer the number of electrically active carriers does not equal the total number of indiffused impurity atoms. The number of atoms is always the larger quantity.

In general, the results showed good reproducibility and conformed to the "rules" of the Van der Pauw technique. Some confidence may therefore be attached to them. A combination of all the work gives some good absolute figures together with an obvious trend towards lower carrier concentrations than initially might be expected. This established, it is necessary to discuss the possible reasons for this effect. A similar situation occurred with our four-point probe measurements [11] 
TABLE III: Hall Effect measurements on the $\mathrm{H} 4$ specimen.

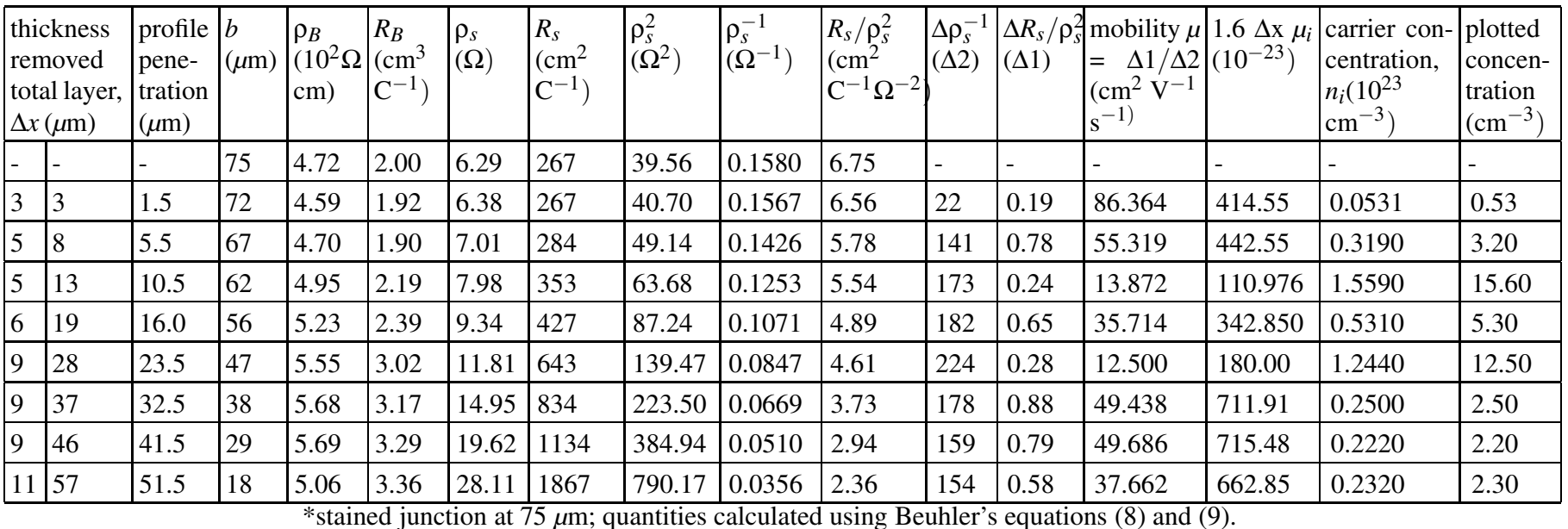

and possible reasons for the observed discrepancy were fully discussed. Of course the same arguments apply to the Hall Effect study.

In conclusion to this section, it is worthwhile looking at the validity of the "one-carrier" type of analysis employed in the Hall Effect calculations. The use of this type of analysis can be open to some question when dealing with III-V compounds because of the large difference between the mobilities of electrons and holes found in these materials. For InP, for example, $\mu_{e} / \mu_{h}$ is about 100 . This kind of situation can lead to the electrons being the dominant species although existing in lower concentrations than the holes. As the "two-carrier" equation for Hall Coefficient, $R_{H}$, demonstrates, a sample may appear n-type due to the multiplying factor $\left(\mu_{e} / \mu_{h}\right)^{2}$ when in fact $p>n$. This equation is,

$$
R_{H}=\frac{1}{e} \frac{\left(p-n b^{2}\right)}{(p+n b)^{2}}
$$

where $b=\mu_{e} / \mu_{h}$.

However, for the type of samples used in these experiments, the doping level is so high that the number of free electrons remaining in the p-type region is necessarily minor, according to the equation,

$$
n p=n_{i}^{2}
$$

The intrinsic carrier concentration, $n_{i}$, for InP at room temperature is $\sim 8 \times 10^{8} \mathrm{~cm}^{-3}$. The only danger area for this approach in diffused crystals with concentration gradients is in the regions for which $N_{a}$ is approximately equal to $N_{d}$. Then the Fermi-Level is near to the centre of the forbidden gap and

$$
n \sim p \sim n_{i}
$$

In this situation, the dominant species would be determined by the mobility values. The regions over which this kind of condition will occur are very narrow and should not affect the bulk of the diffused region. For the previously described homogeneously doped specimens, of course, $N_{a}$ will always be greater than $N_{d}$ and the above situation will never arise. Therefore, the use of "one-carrier" equations is fully justified.

\section{CONCLUSION}

Carrier profiles were obtained using Hall Effect techniques and a wide range of diffusion conditions. These measurements were found to be very satisfactory from an experimental point of view, providing several parameters with good reproducibility.

A typical carrier profile was shown in Fig. 6. After an initial peak, the concentration is followed, away from the surface region, by a uniform level at about $22.5 \times 10^{18} \mathrm{~cm}^{-3}$; its p-n junction, found by chemical staining, corresponds to an impurity atom concentration of $5-6 \times 10^{17} \mathrm{~cm}^{-3}$.

For the multisample experiment, the same general shape of profile was found (Fig. 8) with a uniform level of $4 x$ $10^{18} \mathrm{~cm}^{-3}$ being approached. The stained junction depth corresponds to $9.0 \times 10^{17} \mathrm{~cm}^{-3}$ zinc atoms on the radiotracer profile.

For all of the diffused crystals which were sectioned and probed at successive depths, the transition from $\mathrm{p}$ - to $\mathrm{n}$-type material corresponded fairly closely $(\sim 3 \mu \mathrm{m})$ to the position of the p-n junction as found by staining.

A series of Hall Effect measurements on homogeneously doped specimens gave atom/carrier ratios ranging from 18 to 85. These results are very reliable since the calculation of concentration in this case requires no averaging or sectioning procedures. The diffusions were carried out with a $1 \mathrm{mg} \mathrm{P} / 1$ $\mathrm{mg} \mathrm{Zn}$ source in the temperature range $700-900{ }^{\circ} \mathrm{C}$. The zinc 
atom concentrations varied from $7 \times 10^{19} \mathrm{~cm}^{-3}\left(700^{\circ} \mathrm{C}\right)$ to $3 \times 10^{20} \mathrm{~cm}^{-3}\left(900{ }^{\circ} \mathrm{C}\right)$.

A combined plot of mobility versus carrier concentration for several specimens, including homogeneous doping, was shown in Fig. 7. The general trend is for the mobility to decrease with increasing concentration values. In our next paper on $\mathrm{C}-\mathrm{V}$ measurements we revisit these matters.
[1] E. F. Schubert, Doping in III-V Semiconductors (Cambridge University Press, Cambridge, 2004).

[2] S. Adachi, Properties of Group-IV, III-V and II-VI Semiconductors (Wiley, New York, 2005).

[3] B. Tuck, J. Cryst. Growth 208, 123 (2000).

[4] J. Decobert, D. Herrati, V. Colson, D. Leclerc, and L. Goldstein, J. Cryst. Growth 248, 390 (2003).

[5] K. M. Yu and M. C. Ridgway, Nucl. Instrum. Meth. B 168, 65 (2000).

[6] Y. W. Zhao, Z. Y. Dong, and C. J. Li, J. Cryst. Growth 275, 381 (2005).

[7] M. O. Manasreh, InP and Related Compounds: Materials, Applications and Devices (Wiley, New York, 2000).

[8] S. Aytaç and A. Schlachetzki, Solid-State Electron. 24, 57 (1981).

[9] R. L. Causley and R. A. Lewis, Physica B 302-303, 327 (2001).
[10] E. H. Rhoderick, III-Vs Review 13, 45 (2000).

[11] C. A. C. Sequeira and D. M. F. Santos, Braz. J. Phys. 39, 1077 (2007).

[12] C. A. C. Sequeira and D. M. F. Santos, Czech J. Phys. 56, 549 (2006).

[13] L. J. Van der Pauw, Phillips Research Report 13, 1 (1958).

[14] R. Jakiela, A. Barcz, E. Wegner, and A. Zagojski, Vacuum 78, 417 (2005).

[15] M. G. Beuhler, Stanford Research Report, SEL-66-064 (1966).

[16] R. L. Petritz, Phys. Rev. 110, 1254 (1958).

[17] P. R. Thornton, The Physics of Electroluminescence Devices (Spon, London, 1967).

[18] J. W. Mayer, O. J. Marsh, G. A. Shifrin, and R. Baron, Can. J. Phys. 45, 4073 (1967). 